\title{
Femtomolar Electrochemical Detection of DNA-targets Using Metal Sulfide Nanoparticles
}

\author{
Jacob A. Hansen, ${ }^{\dagger}$ Rupa Mukhopadhyay, ${ }^{\ddagger}$ Jonas Ø. Hansen, ${ }^{\ddagger}$ and Kurt V. Gothelf ${ }^{\dagger *}$ \\ Center for Catalysis and Interdisciplinary Nanoscience Center (iNANO), Department of \\ Chemistry, Langelandsgade 140, Aarhus University, 8000 Aarhus C, Denmark
}

\section{Experimental procedures}

General procedures. Unless mentioned stock solutions were prepared using triple distilled water. All chemicals were purchased from Sigma/Aldrich and used as received.

DNA-sequences. The DNA-sequences purified by FPLC were purchased from DNATechnology A/S, Aarhus. The 5'-thiolated sequences c1, r1, c2, r2, c3 and r3 were synthesized using a 5'-Thiol-Modifier C6 S-S from Glen research. The free thiols were obtained by reduction of the disulfide-protected nucleotides $(\sim 0,01 \mu \mathrm{mol})$ by treatment with tris(carboxyethyl)phosphine (TCEP) $(4 \mathrm{mg}, 14 \mu \mathrm{mol})$ in $250 \mu \mathrm{L}$ of triple distilled water for 30 min. Purification was preformed by freeze drying and redissolving in $80 \mu \mathrm{L}$ of water followed by chromatography using a Microspin ${ }^{\mathrm{TM}}$ G-25 column from Amersham Biosciences. The sequences listed in Table S1 were used without further purification.

Table S1. DNA-sequences

\begin{tabular}{|c|c|c|}
\hline name & $\begin{array}{l}\text { Used in } \\
\text { conjugation } \\
\text { with }\end{array}$ & Sequence $^{\mathrm{a}}$ \\
\hline c1 & Au surface & HS-C $_{6}$-TTTTTTTTTTTTTTTTTTTTTGAAGTCAACCAGCA \\
\hline r1 & $\mathrm{CdS}$ & HS-C ${ }_{6}$-TTTTTTTTTTTTTTTTTTTTTGCTGGTTGACTTCA \\
\hline t1 & - & TGAAGTCAACCAGCAAAAAA \\
\hline c2 & Au surface & HS-C $_{6}$-TTTTTTTTTTTTTTTTTTTTAATCCAGGATCAACA \\
\hline r2 & $\mathrm{ZnS}$ & HS-C $_{6}$-TTTTTTTTTTTTTTTTTTTTTGTTGATCCTGGATT \\
\hline $\mathbf{t 2}$ & - & AATCCAGGATCAACAAAAAA \\
\hline C3 & Au surface & 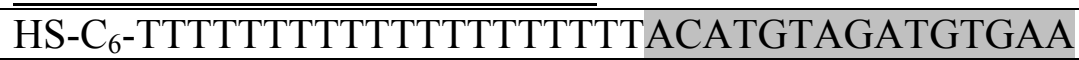 \\
\hline R3 & $\mathrm{PbS}$ & HS-C $_{6}$-TTTTTTTTTTTTTTTTTTTTTTCACATCTACATGT \\
\hline T3 & - & ACATGTAGATGTGAAAAAAA \\
\hline
\end{tabular}

${ }^{\mathrm{a}}$ Complementary capture (c) and reporter (r) sequences are highlighted in grey and complementary sequences of reporter $(\mathbf{r})$ and target $(\mathbf{t})$ sequences are underlined.

Immobilization of DNA on the gold substrate. The gold substrates were made from a solid gold wire ( $1 \mathrm{~mm}$ diameter, Dansk Aedelmetal) flattened to $3 \mu \mathrm{m}$ using a roller, and cut to the desired size of approximately $1 \mathrm{~cm}^{2}$ total surface area. Before generation of the monolayer, the gold substrates were thoroughly cleaned using a Piranha solution. (3:1 of conc. $\mathrm{H}_{2} \mathrm{SO}_{4}: 30 \% \mathrm{H}_{2} \mathrm{O}_{2}$. (NOTE: Great care should be taken, as the solution reacts violently with organic materials.) Immobilization of the 5'-thiol modified nucleotide sequences on gold was performed by covering the gold substrate with solution of the oligonucleotide or mixture of oligonucleotides $(20 \mu \mathrm{L}, 0.0026 \mu \mathrm{mol})$ in $500 \mu \mathrm{L}$ of water and stirring for 24 hours. The 


\section{SUPPORTING INFORMATION}

modified gold substrate was washed twice with water $(2 \times 500 \mu \mathrm{L})$ and then treated with a hexanethiol solution $(1.0 \mathrm{M}$ in $1: 1 \mathrm{v} / \mathrm{v}$ EtOH/Water, $250 \mu \mathrm{L})$ for 1 hour and subsequently washed twice with water $(2 \times 500 \mu \mathrm{L})$.

Preparation of metal sulfide nanoparticles. Nanoparticles of zinc sulfide, lead sulfide and cadmium sulfide were prepared using reverse micelles. ${ }^{1,2}$ For the preparation of each of the three types of nanoparticles, a surfactant emulsion containing bis(2-ethylhexyl)sulfosuccinate (AOT) $(14.0 \mathrm{~g}, 31.5 \mathrm{mmol})$ in a hexane/water mixture $(196 \mathrm{~mL} / 4 \mathrm{~mL})$ was prepared and the emulsion was separated into two subvolumes of $80 \mathrm{~mL}$ and $120 \mathrm{~mL}$ respectively.. The respective metal nitrate $(0.48 \mathrm{~mL}, 1.0 \mathrm{M})$ was added to the $120 \mathrm{~mL}$ subvolume, and $\mathrm{Na}_{2} \mathrm{~S}$ $(0.32 \mathrm{~mL}, 1.0 \mathrm{M})$ to the $80 \mathrm{~mL}$ subvolume. After $1 \frac{1}{2}$ hours of thorough stirring, the two subvolumes were mixed and stirred for additional 1 hour under argon. The nanoparticles formed were subsequently modified by the addition of cysteamine $(0.34 \mathrm{~mL}, 0.32 \mathrm{M}$ in water) and 2-mercaptoethanesulfonic acid $(0.070 \mathrm{~mL}, 3.0 \mathrm{M}$ in water) followed by stirring under argon for 24 hours. The cysteamine/2-mercaptoethansulfonic acid metal sulfide nanoparticles were then obtained by evaporation in vacuo, followed by washing with pyridine, hexane, acetone and methanol. The washing procedure was performed by addition of a solvent to the AOT-quantum dot mixture, followed by centrifugation at $13.000 \mathrm{rpm}$ in $30 \mathrm{~min}$ and removal of the washing liquid. The capped quantum dots where collected after the final washing with methanol and dried in vacuo to give the nanoparticles as powders.

Preparation of nanoparticle-DNA conjugates. A solution of the metal sulfide nanoparticle of choice $(0.5 \mathrm{mg} / \mathrm{mL}, 50 \mu \mathrm{L})$ and the respective 5 '-thiolated nucleotide $(0,001 \mu \mathrm{mol})$ was stirred for 24 hours. The resulting metal sulfide-DNA conjugate was brought to a $0.24 \mathrm{M}$ $\mathrm{NaCl}$ and $0.1 \mathrm{M}$ phosphate buffer $(\mathrm{pH}=7.4)(1 \mathrm{~mL})$ and dialyzed for 48 hours against $0.2 \mathrm{M}$ $\mathrm{NaCl}$ and $0.1 \mathrm{M}$ phosphate buffer $(\mathrm{pH}=7.4)$ containing $0.01 \%$ sodium azide.

The hybridization assay. The hybridization was carried out by addition of the nanoparticleDNA conjugate or mixtures of conjugates to the nucleotide-covered gold substrate in a 500 $\mu \mathrm{L}$ solution of $\mathrm{NaCl}(0.75 \mathrm{M})$ and sodium citrate $(0.15 \mathrm{M})$. This mixture was heated at $60{ }^{\circ} \mathrm{C}$ and left to cool to room temperature for $1 \frac{1}{2}$ hour. The nanoparticle-DNA conjugates captured by hybridization with the DNA-sequences at the surface were then washed thoroughly with water $(3 \times 500 \mu \mathrm{L})$ and with Tris- $\mathrm{HCl}(0.10 \mathrm{M}, \mathrm{pH}=7.5,3 \times 500 \mu \mathrm{L})$. The quantum dots remaining at the surface were now dissolved by treatment of the gold substrates with a solution of $\mathrm{HNO}_{3}(0.10 \mathrm{M}, 500 \mu \mathrm{L})$ for 1 hour.

The competition assay. The competition assay setup was made by immobilizing CdS-r1 and PbS-r3 on a gold substrate functionalized with sequences $\mathbf{c 1}$ and $\mathbf{c} 3$ according to the method described above. The competition experiments were performed by adding the target sequence $\mathbf{t} 3$ (see main article) or $\mathbf{t} \mathbf{1}$ (see below) and stirring the mixture to at room temperature for 5-6 hours. The gold substrates were washed with water $(3 \times 500 \mu \mathrm{L})$ and Tris- $\mathrm{HCl}(0.10 \mathrm{M}, \mathrm{pH}=$ $7.5,3 \times 500 \mu \mathrm{L})$. The nanoparticles remaining at the surface were dissolved by the addition of $\mathrm{HNO}_{3}(0.10 \mathrm{M}, 500 \mu \mathrm{L})$ and the amount and identity of the dissolved metal ions were determined by stripping voltammetry. 


\section{SUPPORTING INFORMATION}

Anodic stripping voltammetry. The solution containing the dissolved quantum dots were transferred into $2 \mathrm{~mL}$ of acetate buffer $(0.20 \mathrm{M}, \mathrm{pH}=5.6)$ containing $10 \mathrm{ppm}$ of mercury ions (from $\mathrm{Hg}(\mathrm{II})$ acetate). The voltammetric stripping measurements were conducted in a standard electrochemical cell using a homebuild potentiostat. ${ }^{3}$ The three electrode setup consisted of a Pt-wire electrode as the counter electrode, a commercially available $\mathrm{Ag} / \mathrm{AgCl}$ reference electrode (World Precision Instruments, FLEXREF) and an in situ formed mercury drop electrode (on a glassy carbon surface) generated, after pre-treatment for $1 \mathrm{~min}$ at $600 \mathrm{mV}$, by accumulation for $2 \mathrm{~min}$ at $-1400 \mathrm{mV}$. The stripping voltammetry was carried out between $1200 \mathrm{mV}$ and $-200 \mathrm{mV}$ using square wave stripping voltammetry unless mentioned otherwise.

\section{Supplementary stripping voltammograms}

Competition experiments at different 3 concentrations. The data shown in Figure $2 \mathrm{~F}$ in the article were obtained from a series of anodic stripping analyses performed after addition of varying amounts of target DNA t3. The stripping voltammograms that are omitted in the article are shown in Figure S1. The minor additional peaks observed in some of the stripping voltammograms are impurities from traces of other metals.
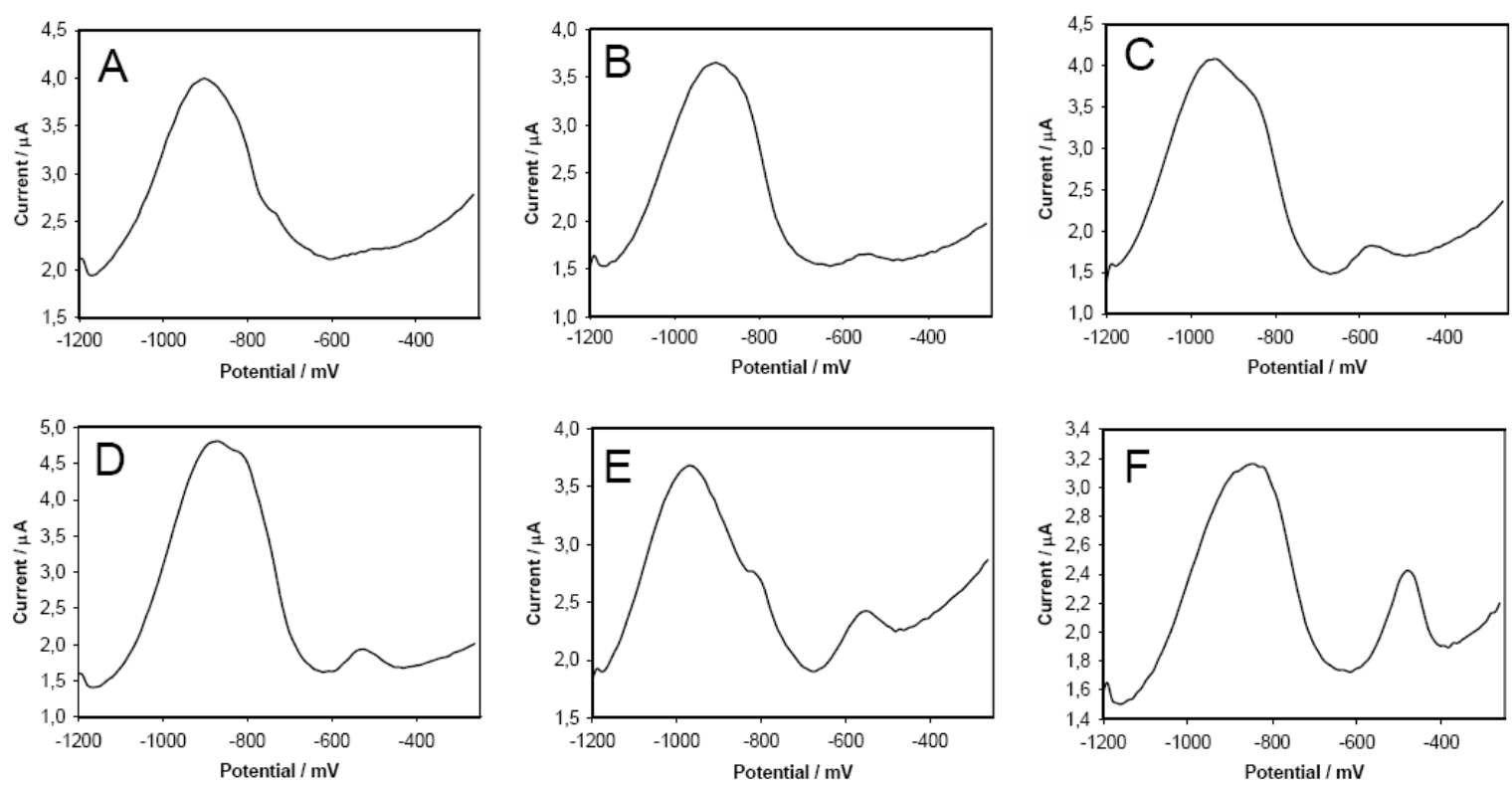

Figure S1. Stripping voltammograms obtained from the competition experiments at different concentrations of t3: (A) 50 fmol, (B) 10 fmol, (C) 5 fmol (D) 1 fmol (E) 0.5 fmol and (F) 0.1 fmol.

Competition experiments with target $\mathbf{t 1}$. In analogy to the competition experiment used for $\mathbf{t} 3$ this competition assay contained CdS-r1 and PbS-r3 immobilized at the gold substrate (Figure $\mathrm{S} 2$ ). The initial ratio between $\mathrm{PbS}$ and $\mathrm{CdS}$ at the surface in this series of experiments, compared to the above described of experiments, is different since different batches of nanoparticles were applied. The stripping voltammograms before and after addition of $\mathbf{t 1}$ (50 


\section{SUPPORTING INFORMATION}

fmol) show that the majority of the CdS particles diffuse from the surface after addition of the target $\mathbf{t} 1$.
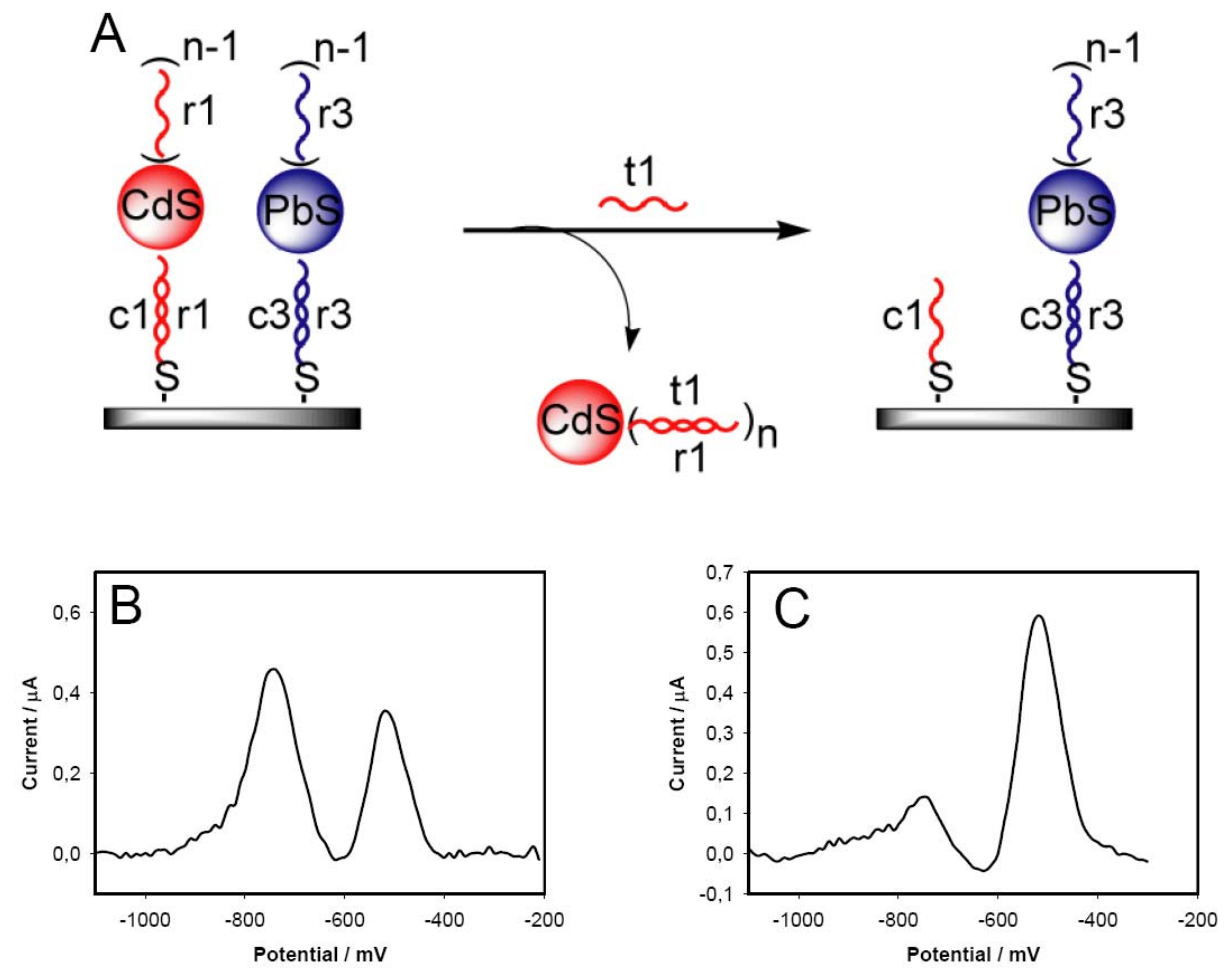

Figure S2. Competition assay for detection of DNA-target t1 (50 fmol in $3 \mathrm{~mL}$ ) (A) and anodic stripping voltammetry analysis of the particles remaining at the surface before (B) and after (C) addition of $\mathbf{t} 1$.

\section{AFM imaging}

Preparation of the sample for characterization of nanoparticle-ssDNA conjugates. The metal sulfide nanoparticle-ssDNA conjugate solutions were prepared by diluting the $1 \mu \mathrm{g} / \mu \mathrm{L}$ stock solution to $1 \mathrm{ng} / \mu \mathrm{L}$ concentration with milliQ water freshly before each imaging experiment. A volume of $5 \mu \mathrm{L}$ of the $1 \mathrm{ng} / \mu \mathrm{L}$ solution was deposited onto freshly cleaved mica substrate and incubated for $30 \mathrm{~min}$ at room temperature in a covered box. The mica piece was then gently washed with $1 \mathrm{~mL}(5 \times 200 \mu \mathrm{L})$ of milliQ water and dried using a soft flow of nitrogen.

Preparation of the sample for competition assay. A flattened gold wire similar to the substrates used for the assay described above was modified first with the ssDNA (c3) and the $\mathrm{C}_{6}$-thiol and then with the PbS-r3 conjugates using the same method as used for the electrochemistry experiments.

(A) Ambient imaging. AFM imaging of CdS-r1 and PbS-r3 on mica was performed in ambient conditions at room temperature $\left(23 \pm 1{ }^{\circ} \mathrm{C}\right)$ using a Veeco equipment with a Nanoscope IIIA controller using mica as the substrate (Figures S3 and S4). All the images were obtained using tapping mode so that the soft metal sulfide-DNA conjugates are least damaged by the scanning tip. Silicon nitride straight spring cantilevers (NSC 35, $\mu$ masch), $110 \mu \mathrm{m}$ long and with the tip curvature radius typically less than $<20 \mathrm{~nm}$, were used within 
the resonance frequency range of $165-240 \mathrm{kHz}$. The tips were used without any modification except that they were cleaned using a UV-tip cleaner (Bioforce Nanosciences, USA) for 30 min prior to imaging. The images were subjected to minimum processing limited to 'brightness and contrast' and 'first order flattening' using the Veeco image processing software. The same tip was used for obtaining all the images considered for making the histograms. The histograms were made from the dimensions along the long axes of the QDssDNA conjugates. Only those particles with well-defined contour were considered for the quantitative analyses.
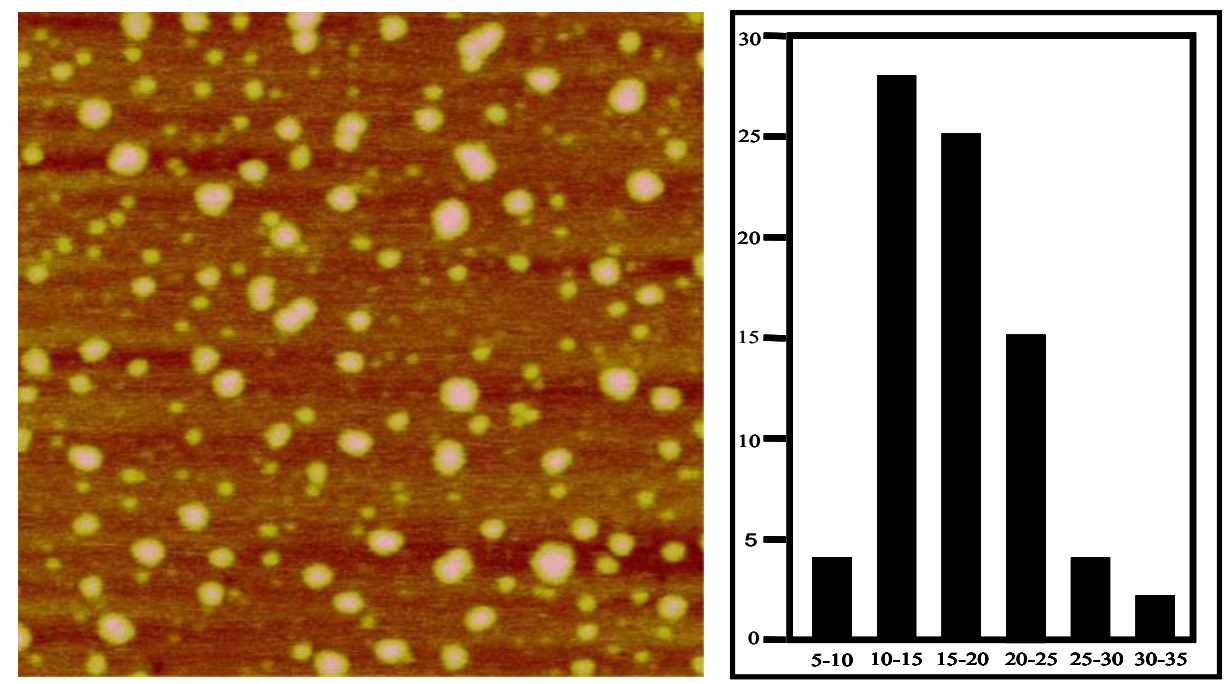

Figure S3: Left: AFM image of PbS-r3 conjugate on mica. Scan range $=550$ x $550 \mathrm{~nm}$. Right: Quantitative dimensional analysis of conjugates.
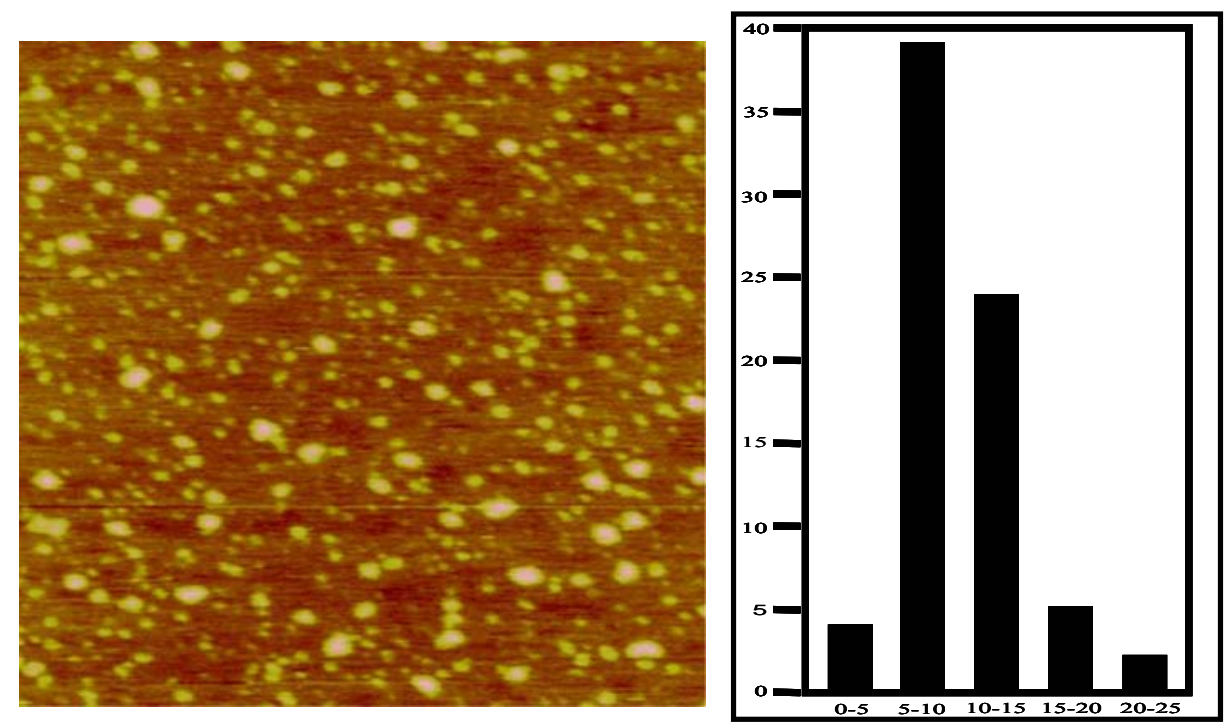

Figure S4: Left: AFM image of CdS-r1 conjugate on mica. Scan range $=440$ x $440 \mathrm{~nm}$. Right: Quantitative dimensional analysis of the conjugates.

(B) Fluid phase imaging. The images were obtained using a Pico SPM equipment (Molecular Imaging) with an AFM 'S' scanner. Imaging was performed at room temperature $\left(23 \pm 1{ }^{\circ} \mathrm{C}\right)$ in situ in real time by the dynamic force mode using magnetically oscillated cantilevers (Magnetic Alternating Current or MAC mode). Type I straight spring silicon MAC 


\section{SUPPORTING INFORMATION}

cantilevers (Molecular Imaging) were used as obtained within a resonance frequency range of $50-90 \mathrm{KHz}$ (in buffer). These cantilevers are about $85 \mu \mathrm{m}$ long and the tip curvature radius is typically less than $10 \mathrm{~nm}$. The tips were cleaned routinely using a UV-tip cleaner (Bioforce Nanosciences, USA) for $30 \mathrm{~min}$ prior to imaging. The fluid cell used for imaging under fluid was routinely cleaned by keeping it in a piranha solution overnight and then washing and boiling in pure water for approximately $30 \mathrm{~min}$ with occasional change of water. Imaging was performed immediately after cleaning the cell with freshly prepared sample in buffered condition ( $0.75 \mathrm{M}$ sodium chloride, $0.15 \mathrm{M}$ sodium citrate). Once the gold substrate modified with PbS-r3 conjugates attached onto the $\mathrm{c} 3$ layer was imaged in the buffered condition, the competing target $\mathrm{t} 3$ was injected and kept for an hour with the tip remaining engaged in the feedback electronics. At the end of this period, the buffer solution was withdrawn and fresh buffer introduced and imaging resumed. The Sscan speed was typically 1-2 lines $\sec ^{-1}$. Minimum processing limited to 'first order flattening' was employed using the Molecular Imaging data analysis software.

\section{References}

1) Willner, I.; Patolsky, F.; Wasserman, J. Angew. Chem. Int. Ed. 2001, 40, 18611864.

2) Wang, J.; Liu, G.; Merkoçi, A. J. Am. Chem. Soc. 2003, 125, 3214-3215.

3) Pedersen, S. U.; Christensen, T. B.; Thomasen, T.; Daasbjerg, K. J. Electroanal. Chem. 1998, 454, 123-143. 